\title{
Design of a SPR sensor for efficacy evaluation of traditional Chinese Medicine
}

\author{
Helin ZHANG ${ }^{1, a}$, Guolong ZHANG ${ }^{1, b}$, Kang ZHANG ${ }^{1, c_{*}}$ \\ ${ }^{1}$ School of computer, Jiangxi University of Traditional Chinese Medicine, Nanchang 330006, China \\ alin_he99@163.com, ${ }^{\mathrm{b}} 65168700 @ q q . c o m,{ }^{\mathrm{c}} 305224190 @ q q . c o m$
}

Keywords: SPR biosensor; traditional Chinese medicine; efficacy evaluation

\begin{abstract}
Objective to antibacterial effect of traditional Chinese medicine for real-time detection, analysis of quantitative efficacy evaluation. Methods the vacuum thermal evaporation plating layer metal film produced fiber SPR sensor, sensing changes in antimicrobial antigen and antibody of each parameter in the process of Chinese medicine, provide the data basis for efficacy evaluation. The results of real-time detection function, objective, accurate, fast and efficient application of the sensor, provides the basis for the evaluation of the antibacterial effect of traditional Chinese medicine quantitative. Conclusion the application of fiber-optic SPR biosensor for antibacterial efficacy evaluation of traditional Chinese medicine is a quantitative basis. Potentially used in guiding the clinical application of traditional Chinese medicine and Chinese medicine accurate evaluation and selection.
\end{abstract}

\section{Introduction}

The bacteria have a serious threat to human health, the majority of bacteria and drug after repeated exposure to the drug sensitivity decline or even disappear, resulting in drug resistance to the efficacy of drug resistance or invalid. Studies have found that a variety of bacteria on a variety of drugs have even reached more than $90 \%$ of drug resistance[1]. In recent years, traditional Chinese medicine has the advantages of a permanent cure, and no side effect, so that people pay more attention to the application of antibacterial drugs. Therefore, in order to guide the clinical use of antimicrobial drugs, the efficacy of the traditional Chinese medicine is effective, rapid and accurate detection and evaluation is a key issue.

At present, the traditional manual detection methods, such as agar dilution method, paper diffusion method, agar screening method[2], will be affected by the factors, the accuracy and accuracy of the method is relatively poor, and the time of bacterial culture is long, and the number of samples is limited. In order to achieve automatic and intelligent real-time detection, we use the SPR biosensor technology, design a set of optical fiber SPR sensor to complete real-time monitoring tasks, to obtain the relevant experimental data, and then determine the drug resistance and to establish an objective evaluation system of the efficacy of antimicrobial drugs.

\section{SPR sensing technology}

SPR sensing technology is a kind of analysis technology based on physical optical properties. The basic principle is that when the incident light is incident on the interface of two different kinds of transparent medium, the reflection light intensity should be the same, but the attenuation caused by the reflection of the metal in the metal, which is caused by the reflection of the metal, which is caused by the reflection of the metal film. The resonance angle changes with the refractive index of the liquid phase, which is directly proportional to the molecular weight of the metal surface, so it can be qualitative and quantitative analysis. This technology provides a fast, non-destructive, direct and accurate detection method, which is a kind of application of the cell level. SPR sensor technology has great application prospect in the evaluation of the antibacterial activity of Chinese herbal medicine.

At present, the SPR sensing system can be divided into 4 types according to its structure, i.e., the prism coupling structure, the diffraction grating structure, the optical waveguide structure and 
the fiber coupling structure. Optical fiber SPR sensor has the advantages of convenient detection of some human being difficult to enter or harmful to the remote sensing and distributed detection through the optical fiber to transmit the sensitive signal in order to realize the remote detection and distributed detection[3]. Optical fiber SPR sensor also has a high detection sensitivity. In this application, the SPR sensor is used to accomplish the real-time detection of the function of Chinese traditional medicine.

\section{Structure design of optical fiber SPR biosensor system}

The SPR biosensor system is composed of four systems, the optical system, the sensor system, the sampling system and the signal detection system.

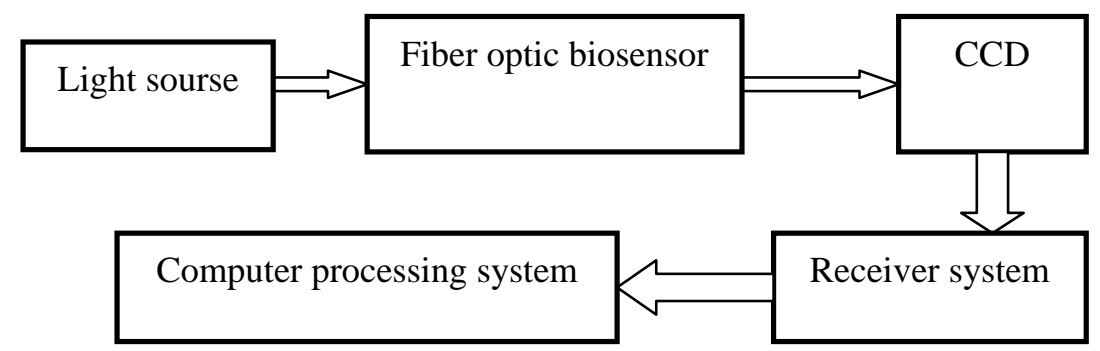

Fig.1. Application system structure diagram

We will be equal to $200 \mathrm{~nm}$ of the ultraviolet light source as the incident light source through the signal input optical system. The sensing system is made of $30 \mathrm{~m}$ quartz optical fiber as the signal transmission channel, and is shown in Figure 2. Into the system design into a $\mathrm{N}$ shaped tube with an effective component of antibacterial Chinese herbal medicine, fiber optic SPR sensor through. Optical fiber SPR biosensor chip was used to carry out the coupling of the bacteria and the substrate. Therefore, the effective molecular solution of the pathogen and the biological identification of the Chinese traditional medicine in the $\mathrm{N}$ - shaped tube is fixed on the surface of the substrate. Finally, the sensitivity of CCD is used to detect the optical signal of the output of the receiving end of the optical fiber, and the intensity parameters of the output light are obtained by the primary amplification of the signal.

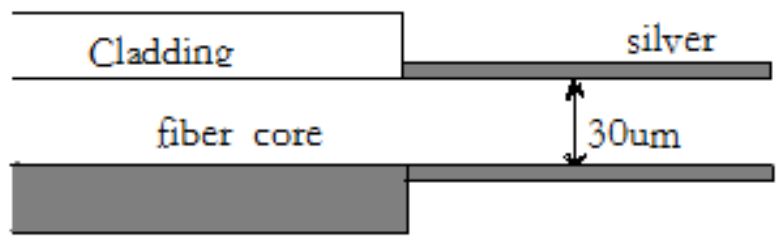

Fig.2. Probe structure

Based on the above idea, the ST fiber SPR sensor probe is fabricated, which takes into account that the metal silver has the best SPR resonance characteristics in all metals, and the design of silver as metal film material[4]. In order to improve the adhesion between silver film and optical fiber, the thickness of the layer of $1 \mathrm{~nm}$ in the surface of quartz fiber material is coated with a layer of chromium.

\section{Application of optical fiber SPR biosensor in the evaluation of the efficacy of antibacterial drugs}

\section{Specific research content and ideas.}

The optical fiber SPR biosensor was designed and fabricated by vacuum thermal evaporation method, and the effect of bacterial culture and antimicrobial activity was analyzed on the sensing chip, and the drug resistance of the bacteria was determined by SPR test.

We use the direct adsorption method to the antigen coupling on the chip surface, when the sample solution containing the analyte (antibody) is used to the surface of the sensor chip, the antigen binding to the surface of the sensor chip and the change of the resonance signal [5]. And 
this change is proportional to the concentration of analyte in the sample. This method is simple and easy to operate, and it is suitable for the measurement of the concentration and molecular weight (>5000) of the material.

\section{Function test of antibacterial Chinese herb}

In the experimental stage, the following 3 questions are mainly to be solved: 1 , the selection of representative bacteria and antibacterial Chinese medicine; 2, the efficacy of the drug resistance and the traditional Chinese medicine were analyzed by using SPR test technology. 3, the feasibility of using SPR in the detection of drug resistance and the efficacy of antibacterial drugs was evaluated.

MRSA infection is a difficult clinical treatment in the treatment of a disease, select the 3 more commonly used antibacterial Chinese herbal medicine (24h), and the role of MRSA in the process of experimental research, through the design of fiber optic SPR biosensor system for its role in the process of drug concentration (MIC) data, and then to its anti MRSA effect is given. The results are as shown in table 1 :

Tab.1. Optical fiber SPR biosensor experimental data of MIC (g/ml) results

\begin{tabular}{cccc}
\hline Chinese Medicine & Chinese rhubarb & Matrine & Coptis \\
\hline After MIC & 0.050 & $>0.100$ & 0.006 \\
\hline
\end{tabular}

These data suggest that rhubarb, flavescent sophora root and Rhizoma Coptidis on MRSA performance varying degrees of antibacterial activity, the Coptis chinensis on MRSA the strongest inhibitory effect, the mic is less than or equal to $0.006 \mathrm{~g} / \mathrm{ml}$, and relatively weak inhibitory effect of rhubarb, Sophora flavescens inhibitory effect was the weakest. The experimental results show that the detection method of the optical fiber SPR biosensor is more fast and real-time.

\section{Key issues in the research}

The key issues are 4: one is to ensure the biocompatibility of fiber SPR biosensor chip and the design of sensor related parameters, so as to ensure the perfect coupling of bacteria in the chip, also makes the experimental data more accurate; two is the bacteria culture, the key is to ensure the normal growth of bacteria. Three is in the sample system to ensure the effective ingredients and the bacteria, and the application system is practical and stable. In the end, it makes the detection system based on the easy to operate the quantitative judgment of the drug resistance, and then gives the data of the evaluation of the efficacy of the antibacterial drugs, and can achieve the purpose of clinical test.

\section{Conclusions}

In this paper, the method of vacuum thermal vapor deposition on quartz optical fiber coated metallic silver film is made of ST type SPR biosensor, which has the characteristics of high sensitivity, real-time and on-line. The experimental data show that it is better to get the relevant parameters in the process of the process, and provide quantitative data for the antibacterial effect of Chinese traditional medicine. Give a new antibacterial efficacy evaluation of traditional Chinese medicine system, Chinese medicine modernization to do what little one can to help.

*Corresponding author: Kang ZHANG, male, College of computer of Jiangxi University of Traditional Chinese Medicine, Nanchang 330006,China; Tel: 18070287029; Email:305224190@qq.com 


\section{References}

[1] Xiao Caide, Sui Senfang. characterization of surface plasmon reso-nance analysis[J].Sensors and Actuators B: chemical 2000 (66): 174177.

[2] Xing Dongming, Han Jingyan, Lu Hong et al. Study on the method to evaluate the efficacy of Chinese medicine on the Journal of Harbin University of Commerce (NATURAL SCIENCE EDITION). 2001 (2): 6 - 4

[3] Sui Senfang, Shonad, Yang Jun. Surface plasmon resonance biosensor [M]. Shanghai: Shanghai science and Technology Press, 2008

[4] Yang Jun, Wang Min, Li Yuanyi et al. Design and implementation of a high precision SPR biosensor (J). Sensor and micro system, 2010,29 (7): 98 - 96

[5] Ma Ruijuan, Miao three. A new method for evaluating the efficacy of traditional Chinese medicine, [J]. 2012,29 (9): 572 - 569 